\title{
Effect of ambient on the thermal parameters of a micromachined bolometer
}

\author{
K. S. Nagapriya* and A. K. Raychaudhuri \\ Department of Physics, Indian Institute of Science, Bangalore 560012, India \\ V. K. Jain, C. R. Jalwania, and Vikram Kumar \\ Solid State Physics Laboratory, Lucknow Road, New Delhi 100054, India
}

\begin{abstract}
The thermal characterization of bolometers is needed for optimal design as well as applications. In this letter, we present results of the effect of environment on the thermal properties of micromachined bolometers. We find that while in vacuum, the thermal response can be represented by a single time constant, in presence of an ambient gas, the thermal response can no longer be described by a single time constant. This will have a direct implication on frequency dependence of responsivity. We present a model to explain our data which involves the finite diffusion time in the ambient gas and the associated extra thermal mass.
\end{abstract}

Microbolometer arrays fabricated using the Microelectromechanical systems technique ${ }^{1}$ are used for thermal image processing. Thermal response characterization of microbolometers is needed for optimal design. Generally, thermal characteristics are quantified through two measurable quantities: the thermal resistance of the bolometer to the substrate $R_{\text {th }}$ and the thermal relaxation time $\tau$. The thermal parameters are measured at a single bias voltage across the bolometer and also as a function of applied bias across the bolometer. ${ }^{2,3}$ Application of excess bias leads to heating of the bolometer. This provides thermal characteristics at elevated temperatures. In this letter, we address the specific issue of ambient. The ambient gas provides an additional thermal link of the bolometer to the substrate in addition to the support hinge and reduces $R_{\mathrm{th}}$. This changes the responsivity of the bolometer, which is $\propto R_{\mathrm{th}}$. By measuring the thermal characteristics in vacuum, air, and helium gas ambient, we have shown that the ambient not only changes the thermal link resistance $R_{\text {th }}$, but more importantly, changes the nature of the time-temperature $(t-T)$ response curve. In vacuum, the $t-T$ curve, on application of a step heat input, shows exponential growth with one time constant, while in air and in He, it shows a significant departure from such a simple behavior. We modeled the performance of the bolometer and have found that the departure from a single time constant arises because a finite mass of the gas ambient becomes associated with the thermal response of the bolometer, giving rise to an extra heat capacity and an associated thermal relaxation time. This changes the frequency response of the bolometer in presence of a $c w$ sinusoidal signal input.

The bolometer used in this investigation consists of an array of $4 \times 4$ elements, each of which is a $\mathrm{Si}_{3} \mathrm{~N}_{4}$ membrane $(50 \mu \mathrm{m} \times 50 \mu \mathrm{m})$ grown on a Si wafer. A scanning electron microscope (SEM) image of a single element is shown in Fig. 1. The elements are connected to the main $\mathrm{Si}$ frame by $\mathrm{Si}_{3} \mathrm{~N}_{4} \quad$ hinges $\quad(5-\mu \mathrm{m}$ width $\times 2-\mu \mathrm{m}$ length $\times 1-\mu \mathrm{m}$ thickness). The thermal element is a Ti heater film $700-\AA$ thick and $4-\mu \mathrm{m}$ wide.

*Electronic mail: ksnaga@physics.iisc.ernet.in
The experiments were carried out in a chamber that can be pumped down to a vacuum of $10^{-5}$ Torr. It can be filled with any desired gas up to a pressure of $1 \mathrm{~atm}$. The thermal characteristics were measured by giving a step power input to the bolometer thermal element (Ti) and recording the temperature response of the bolometer as a function of time, which we call the $(t-T)$ curve. For this we used the Ti element both as a heater and thermometer. To use the Ti element as the thermometer, we calibrated the resistance of the Ti as a function of temperature using a standard platinum thermometer in the temperature range 100 to $350 \mathrm{~K}$. The temperature coefficient of resistance $(T C R)=1 / R(d R / d T)$ $\sim 4 \times 10^{-3} / \mathrm{K}$. The observed calibration is shown in the inset of Fig. 1. The $t-T$ curve was obtained by recording the voltage across the Ti element (which has a constant current bias) using a 16-bit A/D card at a rate of $10-\mathrm{K}$ points/s. Step power input of amplitude $P_{\text {in }}$ leads to a rise in temperature $\Delta T$. In the steady state, $\Delta T=P_{\text {in }} R_{\text {th }}$. To analyze whether the $t-T$ curve is governed by one time constant or not, we have plotted the quantity $\ln \left\{1-\left[T(t)-T_{0}\right] / \Delta T\right\}$ versus $t$, where $T_{0}$ is the temperature of the bolometer in the absence of the power input and is equal to the substrate temperature. When the $t-T$ curve has a single time constant $(\tau)$ the $\ln \{1$ $\left.-\left[T(t)-T_{0}\right] / \Delta T\right\}$ versus $t$ curve is a straight line whose inverse slope gives $\tau$. Any departure from the single $\tau$ behavior will make this curve deviate from a straight line.

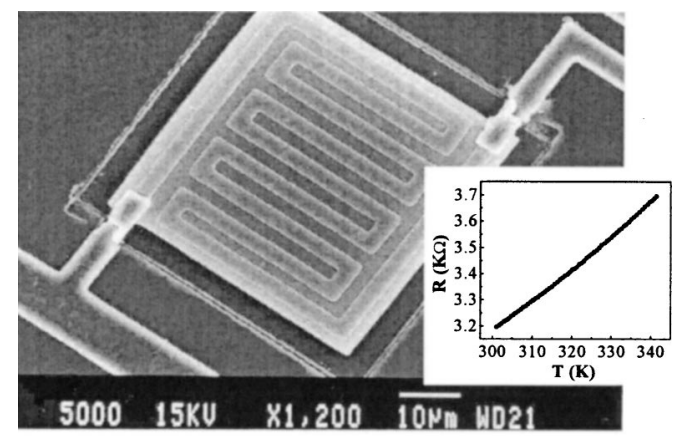

FIG. 1. SEM image of a single bolometer element. The inset shows the temperature dependence of the resistance of the Ti element. 


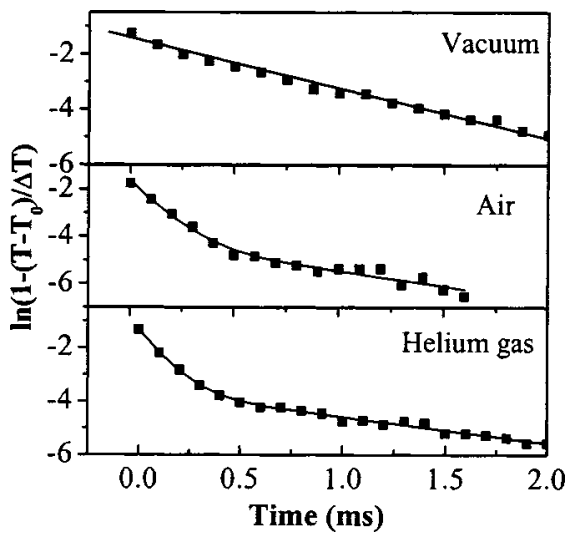

FIG. 2. Thermal response curve of the microbolometers in vacuum, air, and He gas. Lines show fits from the model. Note the single relaxation time behavior of the response in vacuum and two time constants dominated behavior in an ambient gas.

Typical examples of observed $\ln \left\{1-\left[T(t)-T_{0}\right] / \Delta T\right\}$ versus $t$ curves are shown in Fig. 2 for vacuum and with air and helium ambients. In vacuum, the thermal response can be characterized by a single $\tau$, while in air and in He, the curve can be described by a combination of two time constants. From the given $P_{\text {in }}$ and the observed temperature rise $\Delta T$, we could obtain $R_{\text {th }}$ for all the experimental conditions: vacuum, air, and He ambients. The observed $R_{\text {th }}$ is shown in Fig. 3 as function of temperature. In vacuum (pressure $<10^{-3}$ mbar) $R_{\text {th }} \approx 2.5 \times 10^{4} \mathrm{~K} / \mathrm{W}$ at $300 \mathrm{~K}$ and has a shallow temperature dependence. In vacuum, in absence of other thermal pathways, $R_{\text {th }}$ will be determined by conduction through the hinges and the radiation contribution. Absence of a $T^{-3}$-type of a steep temperature dependence in $R_{\text {th }}$ shows that radiation makes a negligible contribution and $R_{\mathrm{th}}$ will be determined by the thermal conductivity $\Lambda$ of the hinge material. From the hinge dimension and the observed $R_{\text {th }}$, we calculate $\Lambda \sim 30 \mathrm{~W} / \mathrm{mK}$, which is the same as the bulk value. In air and in $\mathrm{He}$, there is a large reduction in $R_{\mathrm{th}}$ because the air and $\mathrm{He}$ ambients provide additional thermal links. Understandably, $\mathrm{He}$, which has higher thermal conductivity, shows a lower $R_{\mathrm{th}}$. Thus, in gas environment, it is the gas that determines the value of $R_{\text {th }}$.

Next, we analyze the observed thermal response curve shown in Fig. 2 using a simple model. The basic physics of the model is that the presence of an ambient gas not only provides a thermal link (thus changing $R_{\mathrm{th}}$ ), but also pro-

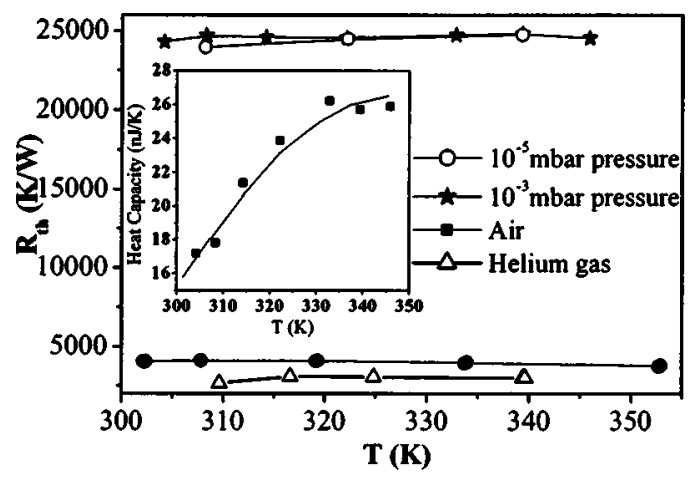

FIG. 3. The thermal resistance $R_{\text {th }}$ in vacuum, air, and He gas as measured from the steady temperature rise $\Delta T$ and $P_{\text {in }}$. The inset shows the heat capacity of the membrane as determined from our experiment.

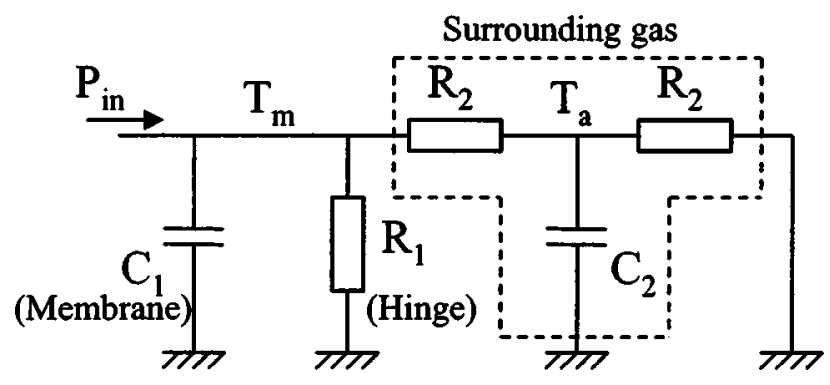

FIG. 4. A thermal model of the bolometer in presence of an ambient.

vides a thermal mass, the heat diffusion through which has a finite time that will lead to additional time constants to the thermal response of the bolometer. The model is outlined subsequently (see Fig. 4). To simplify, we have used the approach of a "lumped circuit" model, in which the thermal masses are represented as capacitors and the thermal resistances are shown as electrical resistors. This is less rigorous than the actual solution of the heat diffusion equation, but it is simple to solve and it captures the essential physics. In Fig. 4, $T_{m}\left[=T(t)-T_{0}\right]$ is the temperature of the membrane with respect to bath (substrate) temperature $T_{0} . T_{a}$ is the average temperature of air with respect to the bath. $R_{1}$ is the thermal resistance of the hinge and $C_{1}$ is the heat capacity of the membrane and hinge combination. $C_{2}$ is the heat capacity of ambient gas that couples to the bolometer and $R_{2}$ is the thermal link due to the gas. We have taken the thermal links of gas to membrane and gas to substrate both as $R_{2}$ for simplicity without sacrificing any feature. The heat balance equations are

$$
\begin{aligned}
& P_{\text {in }}(t)=\frac{T_{m}-T_{a}}{R_{2}}+C_{1} \frac{d T_{m}}{d t}+\frac{T_{m}}{R_{1}}, \\
& \frac{T_{m}-T_{a}}{R_{2}}=\frac{T_{a}}{R_{2}}+C_{2} \frac{d T_{a}}{d t} .
\end{aligned}
$$

For a step heating, $P_{\text {in }}(t)=0$ for $t<0$, and $=P_{\text {in }}$ for $t$ $\geqslant 0$. The solution to this set of coupled differential equations gives

$$
T_{m}=P_{1}\left\{1-\exp (-g t)\left[P_{2} \exp (-f t)+P_{3} \exp (f t)\right]\right\},
$$

where

$$
\begin{aligned}
& 2 g=\left[\left(c^{-1}+2\right) / \tau_{2}\right]+1 / \tau_{1}, \\
& 4 f^{2}=\left(1 / c^{2} \tau_{2}^{2}\right)+\left(2 / c \tau_{1} \tau_{2}\right)+\left(1 / \tau_{1}-2 / \tau_{2}\right)^{2}, \\
& P_{1}=2 P_{\text {in }} /\left[C_{1} \tau_{2}\left(g^{2}-f^{2}\right)\right], \\
& P_{2}=[1 /(2 f)]\left[\tau_{2}\left(g^{2}-f^{2}\right) / 2-(g-f)\right], \\
& P_{3}=[1 /(2 f)]\left[(g+f)-\tau_{2}\left(g^{2}-f^{2}\right) / 2\right] .
\end{aligned}
$$

Here, we have defined $\tau_{1}=R_{1} C_{1}, \tau_{2}=R_{2} C_{2}$, and $c$ $=C_{1} / C_{2}$. The model gives two time constants: $(g-f)^{-1}$ and $(g+f)^{-1}$. In vacuum, the thermal resistance $R_{2} \rightarrow \infty$, $R_{\mathrm{th}}=R_{1}$, and the thermal response is controlled by one time constant $\tau_{1}$. The fits to the data obtained using this model are shown in Fig. 2 as solid lines. In the case of the gas ambient, the response curve with two time constants (Eq. 3) fits the observed data very nicely over the complete range of $t$ over which data are taken. Typical $\tau_{1}$ at $T=300 \mathrm{~K}$ is $\approx 0.5$ 
TABLE I. Thermal parameters obtained from fits to the experimental data.

\begin{tabular}{lcccccc}
\hline \hline Ambient & $\tau_{1}(\mathrm{~ms})$ & $R_{1}(\mathrm{~K} / \mathrm{W})$ & $C_{1}(\mathrm{~nJ} / \mathrm{K})$ & $\tau_{2}(\mathrm{~ms})$ & $R_{2}(\mathrm{~K} / \mathrm{W})$ & $C_{2}(\mathrm{~nJ} / \mathrm{K})$ \\
\hline Vacuum & 0.53 & $2.5 \times 10^{4}$ & 20 & $\infty$ & $\infty$ & $\ldots$ \\
Air & 0.53 & $2.5 \times 10^{4}$ & 20 & 0.37 & $4.0 \times 10^{3}$ & 155 \\
Helium & 0.53 & $2.5 \times 10^{4}$ & 20 & 0.40 & $3.1 \times 10^{3}$ & 222 \\
\hline \hline
\end{tabular}

ms. From the observed value of $R_{1}$ (obtained from the steady-state value of $\Delta T$ and known value of $P_{\text {in }}$ ), we could find the heat capacity $C_{1}$ of the bolometer as a function of $T$ for the temperature range studied (Fig. 3 inset). $C_{1}$ has the main contribution from the bulk of the heat capacity of the bolometer and approximately $1 / 3$ the heat capacity of the hinges. Using this, we obtain a specific heat of $\approx 1.95 \mathrm{~J} / \mathrm{g} \mathrm{K}$ for the material of the bolometer and the hinge. This is much larger than the value of specific heat of $\approx 1 \mathrm{~J} / \mathrm{g} \mathrm{K}$ for $\mathrm{Si}_{3} \mathrm{~N}_{4}$. A value of $\sim 1.5 \mathrm{~J} / \mathrm{g} \mathrm{K}$ for the specific heat of $\mathrm{Si}_{3} \mathrm{~N}_{4}$ has also been reported. ${ }^{4}$

At steady state, $t \rightarrow \infty$, and $T_{m}=\Delta T=P_{1}$. From the observed fits of the data to Eq. (3), the time constants $g^{-1}$, $f^{-1}$, and constants $P_{2}$ and $P_{3}$ can be found. Using the values of $\tau_{1}, R_{1}$, and $C_{1}$ as observed in the case of the vacuum, we can find out $\tau_{2}, R_{2}$, and $C_{2}$, the parameters for the gas ambient. (In our model, the parameters $\tau_{1}, R_{1}$, and $C_{1}$ depend only on the membrane and hinge material, and are independent of the ambient gas). The parameters $\tau_{2}, R_{2}$, and $C_{2}$ are given in Table I.

The heat capacity $C_{2}$ is due to the extra thermal mass of gas around the bolometer through which the heat diffuses over the time scale of one to few $\tau_{2}$. The volume of the gas calculated from the heat capacity is $\sim 1.34 \times 10^{-10} \mathrm{~m}^{3}$ for air and $\sim 2.34 \times 10^{-10} \mathrm{~m}^{3}$ for He. If the volume in which the heat diffuses is taken to be a hemisphere (since the cavity below the membrane $\sim 2 \mu \mathrm{m})$, its radius $\left(r_{g}\right) \approx 400 \mu \mathrm{m}$ for air and $480 \mu \mathrm{m}$ for He. $r_{g}$ thus should be comparable to the thermal diffusion length $L_{D}$ of the gas. The $L_{D}$ is estimated from the relation $L_{D} \approx \pi\left(\tau_{2} D\right)^{0.5}$, where $D$ is the diffusivity of the gas. From the standard $D=5 \times 10^{-5} \mathrm{~m}^{2} \mathrm{~s}^{-1}$ for air and $D=1.1 \times 10^{-4} \mathrm{~m}^{2} \mathrm{~s}^{-1}$ for He, we obtain $L_{D} \approx 440 \mu \mathrm{m}$ for air and $\approx 650 \mu \mathrm{m}$ for He. It can be seen for both the gases, $r_{g} \sim L_{D}$. This is a good validation of the essential physics used in the simple model. In a previous report, it was shown that the $R_{\text {th }}$ increases as the ambient is pumped and the vacuum is reached. ${ }^{4}$ However, the issue of two relaxation times has not been addressed. It may be appreciated that the deviation from a single relaxation time $(\tau)$ dominated thermal response as observed in a gas ambient will severely change the often used expression for the responsivity, $R(\omega) \propto\left(R_{\mathrm{th}} / \sqrt{1+\omega^{2} \tau^{2}}\right)$ which is strictly valid when the bolometer thermal response can be characterized by a single $\tau$.

To summarize, in this letter, we studied the effect of ambient gas on the thermal response of a microbolometer. We find that, while in vacuum, the thermal response can be characterized by a single time constant, the presence of ambient gas changes the nature of the thermal response and it needs two time constants to describe it. With a simple model, we could connect the observed behavior to the physical parameters of the ambient gas.

${ }^{1}$ R. A. Wood, Proc. SPIE 2020, 322 (1994).

${ }^{2}$ X. Gu, G. Karunasiri, G. Chen, U. Sridhar, and B. Xu, Appl. Phys. Lett. 72, 1882 (1998).

${ }^{3}$ P. Neuzil and T. Mei, Appl. Phys. Lett. 80, 1838 (2002).

${ }^{4}$ P. Eriksson, Jan Y. Andersson, and G. Stemme, J. Microelectromech. Syst. 6, 55 (1997) 